\title{
Criminologie
}

\section{Incidence de 1 abus et la négligence envers les enfants : recension des écrits}

\author{
Marc Tourigny et Chantal Lavergne
}

Volume 33, numéro 1, printemps 2000

La victimologie : quelques enjeux

URI : https://id.erudit.org/iderudit/004730ar

DOI : https://doi.org/10.7202/004730ar

Aller au sommaire du numéro

\section{Éditeur(s)}

Les Presses de l'Université de Montréal

ISSN

0316-0041 (imprimé)

1492-1367 (numérique)

Découvrir la revue

Citer cet article

Tourigny, M. \& Lavergne, C. (2000). Incidence de l abus et la négligence envers les enfants : recension des écrits. Criminologie, 33(1), 47-72.

https://doi.org/10.7202/004730ar

\section{Résumé de l'article}

Les mauvais traitements envers les enfants représentent une préoccupation importante dans notre société. Les sévices que subissent les enfants peuvent prendre différentes formes dont celles de l'abus sexuel, l'abus physique, l'abus émotionnel et la négligence. Les études portant sur l'incidence des mauvais traitements envers les enfants s'avèrent un moyen indispensable pour documenter non seulement l'ampleur du phénomène, mais aussi la nature des diverses formes de mauvais traitements, les caractéristiques des victimes, des personnes qui maltraitent et de leur milieu de vie. Ces connaissances sont essentielles au développement des politiques sociales, à l'organisation des services et au développement d'interventions et de pratiques cliniques adaptées aux besoins des familles touchées par ce problème.

Cet article a pour but de faire le point sur les connaissances actuelles concernant l'incidence des mauvais traitements envers les enfants. Plus spécifiquement, il vise : 1) à déterminer les taux d'incidence des diverses formes de mauvais traitements envers les enfants; 2) à examiner l'évolution des taux de mauvais traitements au cours des dernières années; et 3) à cerner les principales limites méthodologiques de ce champ d'études de manière à identifier les défis à relever pour parvenir à une mesure fiable de l'incidence des mauvais traitements envers les enfants.

Quatre méthodes de repérage ont été utilisées pour localiser les publications présentant des taux d'incidence, soit : une recherche bibliographique auprès des principales banques de données informatisées, l'utilisation de recensions d'écrits existantes, une recherche sur le site Web de certains organismes pertinents et finalement, des contacts personnels auprès de chercheurs impliqués dans ce champ d'études. Des critères d'inclusion ont été utilisés afin de sélectionner les publications, ce qui a conduit à l'identification de 24 publications dont un certain nombre présentait des taux d'incidence conduit à l'identification de 24 publications dont un certain nó

Les résultats montrent une variation importante des taux d'incidence, et ce, tant pour les taux de mauvais traitements signalés (avant évaluation par les services de protection de l'enfance) que pour les taux de mauvais traitements jugés fondés après évaluation. Les taux d'enfants signalés varient de 5 à 72 pour 1000 enfants de la communauté alors que les taux d'enfants victimes varient de 0,2 à 110 pour 1000 La négligence représente plus de la moitié des cas de mauvais traitements, suivie des abus physiques (environ $20 \%$ des situations), des abus sexuels (environ $10 \%$ ) et des abus émotionnels (environ $6 \%$ ). L'examen des taux par région montre des différences importantes. De façon générale, les taux d'incidence d'enfants signalés et les taux de signalements fondés aux États-Unis sont de deux à trois fois plus élevés qu'en Australie ou au Québec, mais seulement légèrement plus élevés qu'en Ontario. Les taux de négligence, d'abus sexuel et d'abus physique suivent la même tendance, mais non les taux d'abus émotionnel. Ces variations s'expliquent d'une part par des différences régionales, mais d'autre part par les caractéristiques méthodologiques des études. Les études réalisées à partir des données des agences de protection rapportent des taux beaucoup moins élevés que les études réalisées à partir de données colligées auprès des professionnels de la communauté.

Finalement, les taux d'incidence d'enfants signalés pour mauvais traitements et de signalements jugés fondés ont augmenté de façon importante dans les vingt dernières années. Depuis le début des années 1990, les taux semblent se stabiliser lorsqu'on examine les données provenant des services de protection. Par contre, les taux de mauvais traitements provenant des professionnels de la communauté augmenten durant cette période, et ce, quelle que soit la forme de mauvais traitements considérée.

La discussion des résultats porte sur trois aspects : 1) les facteurs susceptibles d'expliquer la variation importante des taux ; 2) la stabilité des taux d'incidence provenant des études réalisées à partir des données des services de protection comparée à l'augmentation constante des taux d'incidence d'enfants maltraités établis à partir des données recueillies auprès des professionnels de la communauté ; et 3) les limites méthodologiques actuelles et les améliorations à apporter dans les recherches futures.
Ce document est protégé par la loi sur le droit d'auteur. L’utilisation des services d’Érudit (y compris la reproduction) est assujettie à sa politique d'utilisation que vous pouvez consulter en ligne.

https://apropos.erudit.org/fr/usagers/politique-dutilisation/ 


\title{
Incidence de l'abus et la négligence envers les enfants : recension des écrits
}

\author{
Chantal Lavergne \\ Étudiante port-doctorat Groupe de recherches et \\ d'action sur la victimisation des enfants (GRAVE) et \\ Institut de recherche pour le développement social des jeunes (IRDS) • Canada \\ clavergne@mtl.centresjeunesse.qc.ca
}

Marc Tourigny

professeur et chercheur

Département de psychoéducation

Université du Québec à Hull • Canada

Partenariat en recherche

et en intervention en matière d'abus sexuel

envers les enfants (PRIMASE)

Marc_Tourigny@uqah.uquebec.ca

RÉSUMÉ - Les mauvais traitements envers les enfants représentent une préoccupation importante dans notre société. Les sévices que subissent les enfants peuvent prendre différentes formes dont celles de l'abus sexuel, l'abus physique, l'abus émotionnel et la négligence. Les études portant sur l'incidence des mauvais traitements envers les enfants s'avèrent un moyen indispensable pour documenter non seulement l'ampleur du phénomène, mais aussi la nature des diverses formes de mauvais traitements, les caractéristiques des victimes, des personnes qui maltraitent et de leur milieu de vie. Ces connaissances sont essentielles au développement des politiques sociales, à l'organisation des services et au développement d'interventions et de pratiques cliniques adaptées aux besoins des familles touchées par ce problème.

Cet article a pour but de faire le point sur les connaissances actuelles concernant l'incidence des mauvais traitements envers les enfants. Plus spécifiquement, il vise : 1 ) à déterminer les taux d'incidence des diverses formes de mauvais traitements envers les enfants ; 2) à examiner l'évolution des taux de mauvais traitements au cours des dernières années; et 3 ) à cerner les principales limites méthodologiques de ce champ d'études de manière à identifier les défis à relever pour parvenir à une mesure fiable de l'incidence des mauvais traitements envers les enfants. 
Quatre méthodes de repérage ont été utilisées pour localiser les publications présentant des taux d'incidence, soit : une recherche bibliographique auprès des principales banques de données informatisées, l'utilisation de recensions d'écrits existantes, une recherche sur le site Web de certains organismes pertinents et finalement, des contacts personnels auprès de chercheurs impliqués dans ce champ d'études. Des critères d'inclusion ont été utilisés afin de sélectionner les publications, ce qui a conduit à l'identification de 24 publications dont un certain nombre présentait des taux d'incidence provenant de banques de données couvrant plusieurs années.

Les résultats montrent une variation importante des taux d'incidence, et ce, tant pour les taux de mauvais traitements signalés (avant évaluation par les services de protection de l'enfance) que pour les taux de mauvais traitements jugés fondés après évaluation. Les taux d'enfants signalés varient de 5 à 72 pour 1000 enfants de la communauté alors que les taux d'enfants victimes varient de 0,2 à 110 pour 1000. La négligence représente plus de la moitié des cas de mauvais traitements, suivie des abus physiques (environ $20 \%$ des situations), des abus sexuels (environ $10 \%$ ) et des abus émotionnels (environ $6 \%$ ).

L'examen des taux par région montre des différences importantes. De façon générale, les taux d'incidence d'enfants signalés et les taux de signalements fondés aux États-Unis sont de deux à trois fois plus élevés qu'en Australie ou au Québec, mais seulement légèrement plus élevés qu'en Ontario. Les taux de négligence, d'abus sexuel et d'abus physique suivent la même tendance, mais non les taux d'abus émotionnel. Ces variations s'expliquent d'une part par des différences régionales, mais d'autre part par les caractéristiques méthodologiques des études. Les études réalisées à partir des données des agences de protection rapportent des taux beaucoup moins élevés que les études réalisées à partir de données colligées auprès des professionnels de la communauté.

Finalement, les taux d'incidence d'enfants signalés pour mauvais traitements et de signalements jugés fondés ont augmenté de façon importante dans les vingt dernières années. Depuis le début des années 1990, les taux semblent se stabiliser lorsqu'on examine les données provenant des services de protection. Par contre, les taux de mauvais traitements provenant des professionnels de la communauté augmentent durant cette période, et ce, quelle que soit la forme de mauvais traitements considérée.

La discussion des résultats porte sur trois aspects : 1 ) les facteurs susceptibles d'expliquer la variation importante des taux ; 2) la stabilité des taux d'incidence provenant des études réalisées à partir des données des services de protection comparée à l'augmentation constante des taux d'incidence d'enfants maltraités établis à partir des données recueillies auprès des professionnels de la communauté ; et 3) les limites méthodologiques actuelles et les améliorations à apporter dans les recherches futures.

ABSTRACT - The mistreatment of children has become a major social concern. The suffering of children occurs in a variety of forms: sexual, physical or emotional abuse, and negligence. Studies that examine the incidence of child mistreatment have become an indispensable tool in documenting its extent, as well as its diverse forms, the characteristics of its victims and their abusers, and their environments. Such knowledge is essential to forming social policies, organizing services, and developing interventions and clinical practices adapted to the needs of families affected by this problem. 
The purpose of this article is to update current knowledge with regard to the incidence of child mistreatment. Specifically, it intends to determine rates of specific forms of mistreatment, to examine the evolution of such rates in recent years, and to identify important methodological shortcomings of studies in this area in order to identify challenges that must be met to obtain a reliable measurement of the extent of child mistreatment.

Four methods of data collection were used to locate publications presenting rates of incidence: a bibliographical search among major data banks, a review of current literature, research among the web-sites of relevant organizations, and personal contacts with researchers working in this field. Literature was selected according to specific criteria for inclusion; 24 relevant publications were identified, a number of which present incidence rates based on a number of data banks and covering several years.

The results demonstrate a significant variation in incidence rates, not only the rates of reported mistreatment (prior to investigation by child protection services) but also the rates of confirmed mistreatment following assessment. Reporting rates varied from 5 to 72 children per 1000 in the community; the child victim rates varied from .2 to 110 children per 1000 . More than half of the situations of mistreatment consisted of negligence, followed by physical abuse (about $20 \%$ ), sexual abuse (about $10 \%$ ) and emotional abuse (about $6 \%$ ).

An examination of regional rates also demonstrated significant differences. In general, the rates of reported as well as confirmed incidents in the United States were two to three times higher than those in Australia or Quebec, but only minimally higher than rates in Ontario. Rates of negligence and sexual or physical abuse followed the same pattern, while rates of emotional abuse were divergent. Differences among rates may to some extent be explained by regional differences, but methodological differences among studies are also important. Studies based on data from child protection agencies report much lower rates than studies based on data collected from professionals working with abused children in the community.

Finally, rates of reported incidents of child mistreatment and rates of confirmed reports have increased significantly during the last twenty years. According to child protection services' data, rates seem to have stabilized since the beginning of the 1990s. According to data provided by community professionals however, the rate of child mistreatment has increased consistently during this period, whatever the form of abuse considered.

The discussion of results examines three aspects: first, factors that may explain significant rate variations; second, the stability of rates reported by child protection services compared with the consistent rate increase reported by community professionals; and finally, current methodological shortcomings and means of improving future research.

RESUMEN - El maltrato infantil constituye una preocupación de primer orden en nuestra sociedad. Las sevicias sufridas por los menores pueden presentar diversas formas, entre ellas el abuso sexual, el abuso físico, el abuso emocional y la negligencia. Los estudios sobre la incidencia de este problema en los niños pasa a ser un recurso indispensable para documentar no sólo la amplitud del fenómeno, sino también la naturaleza de sus diversas modalidades, las características de las víctimas, las de las 
personas que maltratan y el entorno de sus vidas. Estos conocimientos resultan esenciales cuando se piensa en el desarrollo de las políticas sociales, en la organización de los servicios y en el desarrollo de las intervenciones y las prácticas clínicas acordes con las necesidades de las familias afectadas por el problema.

El presente artículo tiene por finalidad precisar el estado actual de los conocimientos en lo que respecta a la incidencia del maltrato infantil. Más claramente, el intenta: 1) determinar las tasas de incidencia de las diversas formas de maltrato infantil; 2)examinar la evolución de las tasas de este tipo de maltrato en el curso de los últimos años; 3) identificar las principales limitaciones metodológicas presentes en este campo, para hacer frente a los desafíos futuros, de manera que pueda lograrse una medición confiable de la incidencia de este tipo de maltrato en la sociedad.

El autor utiliza cuatro métodos de búsqueda para localizar las publicaciones que contienen datos sobre tasas de incidencia, es decir: una investigación bibliográfica entre los principales bancos de datos informatizados, el uso de recensiones de escritos existentes, la búsqueda en los sitios web de algunos organismos pertinentes, $y$, finaImente, el contacto personal con diversos investigadores que trabajan este tema. Algunos criterios de inclusión han sido aplicados a fin de seleccionar las publicaciones, lo que le ha permitido identificar 24 publicaciones, algunas de las cuales contienen tasas de incidencia provenientes de bancos de datos a lo largo de varios años.

Los resultados muestran una variación importante de las tasas de incidencia, y ello, tanto en lo que se refiere a las tasas de maltratos denunciados (con anterioridad a la evaluación hecha por los servicios de protección de la infancia) como a las tasas de maltratos considerados como fundados, luego de esta evaluación. Las tasas del primer tipo oscilan entre 5 y 72 por 1000 niños en la comunidad, mientras que las tasas del segundo tipo varían entre .2 y 110 por 1000. La negligencia en particular representa más de la mitad de las situaciones de maltrato, seguida por los abusos físicos (cerca del $20 \%$ de las situaciones), de los abusos sexuales (alrededor del $10 \%$ ) y de los abusos de orden emocional (aproximadamente $6 \%$ ).

El examen de las tasas por región muestra asimismo diferencias importantes. De manera general, las tasas de incidencia por denuncia y las tasas de denuncias fundadas son en Estados Unidos de dos a tres veces más altas que en Australia o en Québec, pero ligeramente superiores a las de 0 ntario. Las tasas de negligencia, de abuso sexual y de abuso físico parecen seguir la misma tendencia, mientras que las tasas en cuanto al abuso emocional constituyen una excepción. Tales variaciones se explicarían, por una parte, en razón de las diferencias regionales, y por otra, debido a las características metodológicas de los distintos estudios. Los estudios llevados a cabo a partir de datos suministrados por las agencias de protección de la infancia reportan generalmente tasas mucho más bajas que los estudios realizados a partir de los datos obtenidos por profesionales en la comunidad.

Por último, las tasas de incidencia de casos denunciados por razón de maltrato y las que corresponden a denuncias de este tipo consideradas con fundamento, han registrado un importante aumento en el curso de los últimos 20 años. Desde comienzos de la década de los ' 90 ', las tasas parecen estabilizarse cuando se analizan los datos provenientes de las agencias de protección. En cambio, las tasas de maltrato suministradas por los profesionales que trabajan en la comunidad han aumentado durante el mismo periodo, independientemente de la forma o del tipo de maltrato. 
La discusión de los resultados engloba tres aspectos : 1 ) los factores susceptibles de explicar las variaciones de las diversas tasas; 2 ) la estabilidad de las tasas de incidencia en estudios hechos a partir de datos suministrados por los servicios de protección infantil, comparada al aumento constante de las tasas de incidencia del maltrato, tal como se observa a partir de los datos recolectados por los profesionales de la comunidad; 3 ) las limitaciones metodológicas actuales y los perfeccionamientos posibles en este orden, con vistas a futuras investigaciones en el campo.

\section{Introduction}

Les sévices que subissent les enfants peuvent prendre différentes formes. Parmi l'ensemble des conduites qui représentent une menace pour le bienêtre des enfants, l'abus et la négligence ${ }^{1}$ de la part des figures parentales constituent les formes les plus courantes de victimisation subie par les enfants (Finkelhor et Dziubia-Leatherman, 1994 : 173-183). Ces mauvais traitements, en particulier la négligence, les mauvais traitements psychologiques, certains types d'abus physique et sexuel, représentent d'ailleurs des formes de sévices qui affectent presque exclusivement les enfants. La dépendance de ces derniers vis-à-vis de ceux et de celles qui sont responsables d'assurer les soins nécessaires à leur développement les rendrait plus vulnérables face à ces formes de victimisation.

Les études portant sur l'incidence ${ }^{2}$ des mauvais traitements envers les enfants s'avèrent un moyen indispensable pour documenter non seulement l'ampleur du phénomène, mais aussi la nature des diverses formes de mauvais traitements, les caractéristiques des victimes et de leur milieu de vie, de même que celles des personnes qui maltraitent ces enfants. Ces connaissances sont essentielles au développement des politiques sociales, à l'organisation des services et au développement d'interven-

1. Les termes « abus et négligence » et « mauvais traitements » sont utilisés dans le texte de manière interchangeable pour désigner l'abus sexuel, physique et émotionnel de même que la négligence.

2. L'incidence des mauvais traitements envers les enfants est généralement définie comme le nombre de nouveaux enfants reconnus ou identifiés (généralement par les services de protection de la jeunesse ou par des professionnels) comme ayant été maltraités au cours d'une période donnée, habituellement une année. L'incidence s'exprime soit en nombre absolu (ex. : 348 enfants ont été maltraités en 1998), soit sous forme de taux annuel (ex. : 3 enfants sur 1000 ont été maltraités en 1998). Comme nous le verrons plus loin, cette définition ne correspond pas toujours à celles qu'on retrouve dans les études, la plupart des études d'incidence n'ayant pas contrôlé si, durant une période donnée, l'enfant maltraité était effectivement un nouveau cas non déjà inclus dans la période à l'étude. 
tions et de pratiques cliniques adaptées aux besoins des familles touchées par ce problème. Sur les plans politique et organisationnel, les études d'incidence peuvent guider les politiques sociales en matière de protection de l'enfance, le développement de programmes de prévention et d'intervention et la distribution des ressources humaines et financières nécessaires pour lutter contre le problème. Sur le plan clinique, ces études permettent d'identifier les besoins des enfants victimes et de leur famille ainsi que ceux des personnes coupables d'abus ou de négligence et de contribuer ainsi au développement de programmes adaptés à ces besoins. Enfin, lorsque ces études sont répétées dans le temps, elles permettent de suivre l'évolution du problème et fournissent des indicateurs sur l'efficacité des services curatifs et préventifs.

L'intérêt pour ce type de connaissances a donné lieu au cours des dernières années au développement de différentes stratégies de recherche. Elles reposent généralement sur l'utilisation de quatre sources de données qui correspondent à quatre niveaux d'identification des enfants victimes de mauvais traitements. Ces quatre niveaux se présentent sous la forme d'une pyramide. Au premier niveau, en haut de la pyramide, on retrouve les cas connus des services de protection, c'est-à-dire les signalements reçus et retenus $^{3}$, de même que ceux considérés, à la suite d'une évaluation, comme fondés et non fondés. Ce premier niveau est souvent considéré comme la pointe de l'iceberg parce que l'ampleur du phénomène y serait sousévaluée. Au second niveau, on retrouve les enfants maltraités qui sont connus des professionnels issus de différents types de services ou d'institutions (par exemple, les hôpitaux, les services sociaux, les écoles, les corps policiers, les tribunaux, etc.). Les enfants identifiés à ce niveau n'ont pas nécessairement été signalés aux services de protection. Le troisième niveau correspond aux enfants maltraités qui sont connus des membres de la communauté où ils vivent (population générale). Ces enfants ne sont pas nécessairement connus des professionnels ou des services de protection. Enfin, les enfants du dernier niveau correspondent aux situations de mauvais traitements qui ne sont pas identifiées comme telles par quiconque (Sedlak, 1991 ; Trocmé, 1992). Cette classification permet de

3. Il est à noter que, dans plusieurs sytèmes de protection de l'enfance dont celui du Québec, ce premier niveau peut également inclure des signalements qui n'ont fait l'objet que d'une évaluation minimale visant à ne retenir que les cas qui s'apparentent aux critères de la loi. Une fois que la recevabilité du signalement est établie, la seconde étape consiste en une évaluation plus en profondeur de la situation signalée dans le but de statuer sur les risques qu'elle comporte pour la sécurité ou le développement de l'enfant. 
rendre compte des liens entre les niveaux d'identification et de mieux comprendre les statistiques produites par les études d'incidence des mauvais traitements envers les enfants.

Il existe quelques recensions d'études d'incidence des mauvais traitements envers les enfants. Ces recensions présentent toutefois certaines limites importantes : 1) la méthodologie des auteurs est rarement explicitée de sorte qu'il est difficile de savoir quels critères ont été utilisés dans la sélection des études épidémiologiques ; et 2) la présentation des résultats de recherche ne semble tenir compte d'aucun critère spécifique, ce qui rend pratiquement impossible une comparaison des études examinées, que ce soit au niveau des résultats produits ou des méthodologies utilisées. Une seule recension (Tonmyr, 1998), présentée comme un répertoire des études d'incidence et de prévalence des mauvais traitements envers les enfants menées à l'échelle internationale, dépasse certaines de ces limites : les stratégies de recherche bibliographique, les bases de données, les sites Web et les organismes consultés sont présentés, la méthodologie de recherche est explicitée et les concepts d'incidence et de prévalence sont définis. Un bref résumé de chacune des études est présenté accompagné d'un tableau sommaire qui rend compte de certains aspects méthodologiques et des principaux résultats de recherche. Toutefois, les critères de sélection des études sont peu explicites et l'analyse qui y est faite est très peu approfondie.

Dans ce contexte, le but poursuivi dans cet article consiste à faire le point sur les connaissances actuelles concernant l'incidence des mauvais traitements envers les enfants. Plus spécifiquement, cette recension a pour objectifs : 1) de déterminer les taux d'incidence des mauvais traitements envers les enfants, et de leurs diverses formes : l'abus sexuel, l'abus émotionnel, l'abus physique et la négligence ; 2) d'examiner l'évolution des mauvais traitements envers les enfants au cours des dernières années ; et 3) d'identifier les principales limites méthodologiques de ce champ d'études de manière à identifier les défis à relever pour parvenir à une mesure fiable de l'incidence des mauvais traitements envers les enfants.

\section{Méthodologie}

Quatre méthodes de repérage ont été utilisées pour localiser les publications sur l'incidence des mauvais traitements. Une recherche bibliographique a été effectuée auprès des principales banques de données (Child Abuse and Neglect, Eric, Medline, Psyclit et Sociofile). Les mots 
clés utilisés dans le cadre de cette recherche sont : incidence, epidemiology, international, survey, frequency. Ces mots clés ont été associés aux termes suivants : sexual abuse, physical abuse, emotional or psychological abuse, child abuse and neglect et child maltreatment. Des recensions d'écrits (Starr et al., 1990 ; D’Antonio et al., 1993 ; Tonmyr, 1998) ont également été utilisées pour repérer les publications qui n'ont pu être identifiées à l'aide de la technique précédente. Une troisième stratégie a consisté à interroger des banques de données accessibles via le site Web de certains organismes pertinents (National Clearinghouse on Child Abuse and Neglect Information, États-Unis ; Statistique Canada, Canada ; National Center for the Protection of Cruelty to Children, États-Unis ; Australian Institute of Health and Welfare, Australie). Finalement, certaines publications ont été identifiées à travers des contacts avec des chercheurs du domaine.

Pour être incluses dans la recension, les publications devaient : 1) être écrites en langue française ou anglaise ; 2) documenter les cas connus des agences de protection ou des professionnels de la communauté (niveaux 1 et 2) ; 3) faire état d'un taux d'incidence, c'est-à-dire d'un nombre d'enfants maltraités par rapport à une population étudiée ${ }^{4}$. Au total, 24 publications ont été retenues dans le cadre de cette recherche bibliographique. Un certain nombre de ces publications présentaient des taux d'incidence provenant de banques de données qui couvrent plusieurs années. Voici une brève description de ces banques.

Aux États-Unis, le U.S. Department of Health and Human Services, en collaboration avec 49 États participants, a créé en 1988 The National Child Abuse and Neglect Data System (NCANDS) qui constitue la principale source d'informations nationale sur les enfants victimes d'abus et de négligence signalés aux organismes et services de protection américains (U.S. Department of Health and Human Services, 1995 ; 1998). Les données tirées du NCANDS sont publiées chaque année depuis 1990. Elles sont recueillies au mois de juillet et versées par chacun des États participants dans un système informatisé. Depuis 1995, une collecte de données concernant les enfants qui font l'objet des signalements évalués est également effectuée auprès des États (11) en mesure de fournir des données par individu. Les représentants des organismes ou des services de protection qui participent à l'étude disposent d'un cahier où sont définis les différents renseignements à colliger et peuvent avoir

4. Les publications qui ne rapportent que le nombre d'enfants identifiés comme victimes d'abus ou de négligence n'ont pas été retenues dans la présente recension. 
accès à une assistance technique en cas de besoin. L'ensemble des données transmises est soumis à une procédure de vérification et de validation. Le nombre d'États américains qui participent à l'étude varie de 31 à 51 selon les années et le type de données colligées.

Le National Committee to Prevent Child Abuse (NCPCA) procède, depuis 1987, à une cueillette d'informations détaillée par téléphone auprès des représentants des organismes de protection situés dans les 50 États et le district de Columbia. Les données recueillies concernent le nombre d'enfants victimes d'abus et de négligence et les changements survenus en matière de législation, de politiques sociales et de financement des services d'aide sociale à l'enfance (McCurdy et Daro, 1994 : 75-94 ; Wang et Daro, 1998). L'organisme publie également des données sur le nombre d'enfants décédés par suite de mauvais traitements de la part des figures parentales. Ces données sont présentement les seules disponibles sur cette question en Amérique du Nord. Le nombre d'États qui participent varie également selon les années et le type de données recueillies.

Le National Incidence Study (NIS) est une enquête nationale portant sur les enfants victimes d'abus et de négligence (NIS-1 : U.S. Department of Health and Human Services, 1981 ; NIS-2 : Sedlack, 1991 ; NIS-3 : Sedlack et Broadhurst, 1996). Les données sont recueillies auprès d'un échantillon représentatif des comtés américains composé d'intervenants issus de divers organismes. La taille de l'échantillon varie d'une étude à l'autre. Le contenu des formulaires d'enquête soumis par les participants concernant chaque enfant identifié comme victime de mauvais traitements est ensuite analysé par l'équipe de recherche afin de déterminer si les situations correspondent aux définitions de l'étude. Trois enquêtes ont été réalisées jusqu'à maintenant, soit en 1979/80 (NIS-1), en 1986 (NIS-2) et en 1993 (NIS-3). Lors de ces trois enquêtes, deux critères de gravité ont été utilisés afin d'identifier les enfants maltraités. Le premier critère (utilisé dans les trois études) est celui des conséquences négatives observables chez les enfants à la suite du mauvais traitement. Le second critère (utilisé dans le NIS-2 et le NIS-3) est celui de la menace que représentent les mauvais traitements pour la santé et la sécurité des enfants. Ces derniers n'ont pas nécessairement subi de conséquences immédiates mais courent un risque important d'en subir éventuellement.

Au Québec, le ministère de la Santé et des Services sociaux tient une banque de données sur les dossiers pris en charge par la Protection de la jeunesse (MSSS, 1995). Il s'agit d'un système d'information conçu 
comme un outil de référence visant à permettre une « compréhension commune des données et des modalités d'échange de celles-ci entre les établissements, les régies régionales et le ministère » (MSSS, 1995 : 2). Le système d'information CJ-LPJ fournit de l'information sur le traitement des signalements retenus (les signalements non retenus ne sont pas versés dans le système). Ce traitement est décrit par une série de variables qui rendent compte des principales décisions prises à chacune des étapes du processus LPJ (réception/traitement des signalements, évaluation/ orientation et révision). L'enfant constitue l'unité de mesure utilisée. Les données sont transmises par chaque centre jeunesse quatre fois par année entre le $1^{\text {er }}$ avril et le 31 mars de l'année suivante. Dans le but d'assurer la validité et la comparabilité des données contenues dans la banque, un cahier présentant les définitions des différents renseignements à colliger est mis à la disposition de chaque centre jeunesse. Des procédures de validation des données sont également appliquées à l'ensemble des données transmises au MSSS. Selon les années, le nombre de centres jeunesse transmettant des données varie de 12 à 15 , sur un total possible de 16. Soulignons que les signalements se rapportant à l'alinéa 38h (troubles de comportement sérieux) de la Loi sur la protection de la jeunesse ont été exclus du calcul des taux pour des raisons de comparabilité avec les autres études.

\section{Caractéristiques méthodologiques des études}

Plusieurs caractéristiques méthodologiques des études ont été documentées dans la recension (voir tableau 1). Une première caractéristique est celle du type de définitions utilisées pour les mauvais traitements, soit les définitions provenant des lois en vigueur (légales) ou les définitions produites par les chercheurs de l'étude. Une seconde est la méthode de collecte des données, selon que celles-ci sont recueillies à partir des systèmes d'information des services de protection (statistiques officielles), de l'examen des dossiers des agences ou par des questionnaires administrés aux intervenants. Une troisième caractéristique précisée est la source des données, soit que celles-ci concernent les situations de mauvais traitements connues des services de protection (niveau 1), soit qu'elles ont trait aux cas identifiés par d'autres professionnels de la communauté (niveau 2). La quatrième caractéristique a trait aux mesures prises par les chercheurs pour éviter la duplication des enfants victimes. Dans certaines études, la duplication a été contrôlée partiellement ou totalement alors que dans d'autres aucune mesure n'a été prise à cet 
égard. La population à l'étude est décrite généralement en termes de situation géographique (pays, comté, province, ville, etc.) et de caractéristiques propres (population générale, groupe ethnique spécifique, familles d'accueil, etc.). L'unité de mesure pour le calcul des taux d'incidence peut être soit les enfants ou les familles (ayant des enfants d'un âge déterminé). La période de collecte est mentionnée en nombre de mois, avec indication des années couvertes par l'étude. Mentionnons en terminant que tous les taux présentés dans le tableau 1 sont des taux annuels d'incidence de mauvais traitements pour 1000 enfants ou familles selon l'unité de mesure.

\section{Une définition des différentes}

formes d'abus et de négligence

Définir l'abus et la négligence constitue un préalable à toute démarche d'analyse de ces problématiques. Actuellement, il n'existe toutefois aucune définition généralement reconnue des mauvais traitements envers les enfants. Celle-ci peut englober plusieurs comportements et omissions, dont l'abus physique, sexuel, émotionnel et la négligence.

Dans le cadre de la présente recension d'écrits, la négligence est envisagée comme un manque chronique d'attention aux besoins de base de l'enfant qui constitue, selon le point de vue de la communauté et du milieu professionnel, un risque significatif pour la santé et le bien-être des enfants, et ce, quelle qu'en soit la cause (Dubowitz et al., 1993). Les besoins de base généralement considérés sont ceux relatifs au gîte, à l'habillement, à la nourriture, aux soins de santé, à l'éducation, à la protection, à la surveillance et aux soins affectifs. Cette forme de mauvais traitements consiste donc en des omissions de la part de ceux qui sont responsables des soins à l'enfant. Quant aux abus, ils sont définis comme des gestes de commission, en général des actes de violence de nature physique, sexuelle ou émotionnelle, qui sont principalement (mais pas uniquement) le fait des personnes responsables de l'enfant et qui sont considérés par la communauté et le milieu professionnel comme dommageables pour leur santé et leur bien-être (Garbarino et Gillian, 1980). L'abus physique comprend des comportements de violence physique comme la raclée, l'isolement excessif, les brûlures, les étouffements, l'utilisation d'une arme ou la menace d'en utiliser une et autres gestes de violence (MSSS, 1991). L'abus sexuel réfere à des relations hétérosexuelles ou homosexuelles entre un enfant et une ou plusieurs personnes en situation de pouvoir qui impliquent un 
T A B LE A U 1

Taux d'incidence annuel des signalements pour mauvais traitements et des signalements jugés fondés après évaluation (toutes formes confondues) et taux d'incidence annuel par forme de mauvais traitements

\begin{tabular}{|c|c|c|c|c|c|c|c|c|c|}
\hline \multirow{2}{*}{$\begin{array}{c}\text { \# Études } \\
\text { Auteur/Année/ } \\
\text { Pays }\end{array}$} & \multicolumn{2}{|c|}{$\begin{array}{l}\text { Méthodologie/ } \\
\text { Échantillon'1 }\end{array}$} & \multicolumn{7}{|c|}{ Taux d'incidence annuel (/1000) } \\
\hline & $\begin{array}{c}\text { Définitions } \\
\text { Méthode / Niveau }\end{array}$ & $\begin{array}{l}\text { Contrôle } \\
\text { Duplication }\end{array}$ & $\begin{array}{l}\text { Population } \\
\text { Unité } \\
\text { Période de } \\
\text { collecte }\end{array}$ & Signalé $^{2}$ & Fondé $^{2}$ & $\begin{array}{l}\text { Ab. } \\
\text { sex. }\end{array}$ & $\begin{array}{l}\text { Ab. } \\
\text { émo. }\end{array}$ & $\begin{array}{l}\text { Ab. } \\
\text { phys. }\end{array}$ & Négl. \\
\hline $\begin{array}{l}\text { \#1 Johnstone \& } \\
\text { Broadbent (1998) } \\
\text { Australie }\end{array}$ & $\begin{array}{l}\text { Déf. légales } \\
\text { Statistiques } \\
\text { Niveau } 1\end{array}$ & Non & $\begin{array}{l}\text { Générale } \\
\text { australienne } \\
\text { Enfants } \\
\text { (0-16 ans) } \\
12 \text { mois } 96 / 97\end{array}$ & $\begin{array}{l}-- \\
--\end{array}$ & $\begin{array}{l}2,1 \text { à } \\
6,2\end{array}$ & $\begin{array}{l}0,3 \mathrm{à} \\
1,2\end{array}$ & $\begin{array}{l}0,9 \text { à } \\
2,5\end{array}$ & $\begin{array}{l}0,7 \text { à } \\
2,5\end{array}$ & $\begin{array}{l}0,2 a ̀ a \\
1,8\end{array}$ \\
\hline $\begin{array}{l}\text { \#2 Broadbent \& } \\
\text { Bentley (1997) } \\
\text { Australie }\end{array}$ & $\begin{array}{l}\text { Déf. légales } \\
\text { Statistiques } \\
\text { Niveau } 1\end{array}$ & Non & $\begin{array}{l}\text { Générale } \\
\text { australienne } \\
\text { Enfants } \\
(0-16 \text { ans) } \\
12 \text { mois } 95 / 96\end{array}$ & 11,6 & 5,8 & 1,0 & 1,8 & 1,7 & 1,3 \\
\hline $\begin{array}{l}\text { \#3 Angus \& Hall } \\
\text { (1996) } \\
\text { Australie }\end{array}$ & $\begin{array}{l}\text { Déf. légales } \\
\text { Statistiques } \\
\text { Niveau } 1\end{array}$ & Non & $\begin{array}{l}\text { Générale } \\
\text { australienne } \\
\text { Enfants } \\
\text { (0-16 ans) } \\
12 \text { mois } 94 / 95\end{array}$ & 13,5 & 6,1 & 1,0 & 1,7 & 1,8 & 1,6 \\
\hline $\begin{array}{l}\text { \#4 Pieterse \& } \\
\text { Van Urk }(1989)^{3} \\
\text { Pays-Bas }\end{array}$ & $\begin{array}{l}\text { Déf. chercheurs } \\
\text { Questionnaire } \\
\text { Niv. } 2 \\
\text { (médecins) }\end{array}$ & Non & $\begin{array}{l}\text { Générale } \\
\text { hollandaise } \\
\text { Enfants } \\
\text { ( } 0 \text {-17 ans) } \\
12 \text { mois } 1983 \\
12 \text { mois } 1974\end{array}$ & $\begin{array}{l}-- \\
--\end{array}$ & $\begin{array}{l}0,7 \\
0,2\end{array}$ & $\begin{array}{l}0,0 \\
0,0\end{array}$ & $\begin{array}{l}0,4 \\
0,0\end{array}$ & $\begin{array}{l}0,1 \\
0,2\end{array}$ & $\begin{array}{l}0,2 \\
0,0\end{array}$ \\
\hline $\begin{array}{l}\text { \#5 Mackenzie et al. } \\
\text { (1993) } \\
\text { Irlande du Nord }\end{array}$ & $\begin{array}{l}\text { Déf. chercheurs } \\
\text { Questionnaire } \\
\text { Niveaux } 1 \text { et } 2\end{array}$ & Oui & $\begin{array}{l}\text { Générale } \\
\text { Irlande du } \\
\text { Nord } \\
\text { Enfants } \\
(0-16 \text { ans }) \\
12 \text { mois } 1987\end{array}$ & -- & -- & 0,8 & -- & -- & -- \\
\hline $\begin{array}{l}\text { \#6 Benedict et al. } \\
(1994)^{4} \\
\text { Etats-Unis }\end{array}$ & $\begin{array}{l}\text { Déf. légales } \\
\text { Statistiques } \\
\text { Niveau } 1\end{array}$ & Non & $\begin{array}{l}\text { Générale } \\
\text { Baltimore } \\
\text { Familles } \\
(0-17 \text { ans) } \\
\text { Fam. accueil } \\
3 \text { ans } 85 / 87\end{array}$ & $\begin{array}{l}13,8 \\
50\end{array}$ & $\begin{array}{r}2,8 \\
11,0\end{array}$ & $\begin{array}{l}1,0 \\
4,3\end{array}$ & $\begin{array}{l}-- \\
--\end{array}$ & $\begin{array}{l}1,8 \\
3,1\end{array}$ & $\begin{array}{l}-- \\
3,6\end{array}$ \\
\hline $\begin{array}{l}\text { \#7 Larson et al. } \\
\text { (1987) } \\
\text { Etats-Unis }\end{array}$ & $\begin{array}{l}\text { Déf. chercheurs } \\
\text { Questionnaire } \\
\text { Niv. } 2 \text { (écoles) }\end{array}$ & Oui & $\begin{array}{l}\text { Migrants } \\
\text { américains } \\
\text { Enfants } \\
\text { (0-17 ans) } \\
12 \text { mois } 1982\end{array}$ & -- & 110,6 & 2,5 & 9,3 & 6,5 & 91,6 \\
\hline $\begin{array}{l}\text { \#8 Sedlack \& } \\
\text { Broadhurst } \\
(1996)^{5} \\
\text { NIS-3, États-Unis }\end{array}$ & $\begin{array}{l}\text { Déf. chercheurs } \\
\text { Questionnaire } \\
\text { Niveaux } 1 \text { et } 2\end{array}$ & Oui & $\begin{array}{l}\text { Générale } \\
\text { américaine } \\
\text { Enfants } \\
\text { (0-17 ans) } \\
3 \text { mois } 1993\end{array}$ & $\begin{array}{l}-- \\
--\end{array}$ & $\begin{array}{l}23,1 \\
41,9\end{array}$ & $\begin{array}{l}3,2 \\
4,5\end{array}$ & $\begin{array}{l}3,0 \\
7,9\end{array}$ & $\begin{array}{l}5,7 \\
9,1\end{array}$ & $\begin{array}{l}13,1 \\
29,2\end{array}$ \\
\hline $\begin{array}{l}\text { \#9 NIS-2 (1991) } \\
\text { États-Unis }\end{array}$ & Idem & Idem & $\begin{array}{l}\text { Idem } \\
3 \text { mois } 1986\end{array}$ & & $\begin{array}{l}14,8 \\
22,6\end{array}$ & $\begin{array}{l}1,9 \\
2,1\end{array}$ & $\begin{array}{l}2,5 \\
3,0\end{array}$ & $\begin{array}{l}4,3 \\
4,9\end{array}$ & $\begin{array}{r}7,5 \\
14,6\end{array}$ \\
\hline $\begin{array}{l}\# 10 \text { NIS-1 } \\
\text { (1979-1980) } \\
\text { Etats-Unis }^{5}\end{array}$ & Idem & Idem & $\begin{array}{l}\text { Idem } \\
3 \text { mois } 79 / 80\end{array}$ & & 9,8 & 0,7 & 2,1 & 3,1 & 4,9 \\
\hline $\begin{array}{l}\text { \#11 Wang \& Daro } \\
\text { (1998) } \\
\text { NCPCA, É.-U. }\end{array}$ & $\begin{array}{l}\text { Déf. légales } \\
\text { Statistiques } \\
\text { Niveau } 1\end{array}$ & Non & $\begin{array}{l}\text { Générale } \\
\text { américaine } \\
\text { Enfants } \\
(0-17 \text { ans })\end{array}$ & & & & & & \\
\hline & & & 12 mois 1997 & 47 & 15 & 1,2 & 0,6 & 3,3 & 8,1 \\
\hline NCPCA & Idem & Idem & 12 mois 1996 & 46 & 15 & -- & -- & -- & -- \\
\hline
\end{tabular}




\section{T A B LE A U 1 (Suite)}

Taux d'incidence annuel des signalements pour mauvais traitements et des signalements jugés fondés après évaluation (toutes formes confondues) et taux d'incidence annuel par forme de mauvais traitements

\begin{tabular}{|c|c|c|c|c|c|c|c|c|c|}
\hline \multirow{2}{*}{$\begin{array}{l}\text { \# Études } \\
\begin{array}{c}\text { Auteur/Année/ } \\
\text { Pays }\end{array}\end{array}$} & \multicolumn{2}{|c|}{$\begin{array}{l}\text { Méthodologie/ } \\
\text { Échantillon }^{1}\end{array}$} & \multicolumn{7}{|c|}{ Taux d'incidence annuel $(/ 1000)$} \\
\hline & $\begin{array}{c}\text { Définitions } \\
\text { Méthode / Niveau }\end{array}$ & $\begin{array}{l}\text { Contrôle } \\
\text { Duplication }\end{array}$ & $\begin{array}{l}\text { Population } \\
\text { Unité } \\
\text { Période de } \\
\text { collecte }\end{array}$ & Signalée ${ }^{2}$ & Fondé $^{2}$ & $\begin{array}{l}\text { Ab. } \\
\text { sex. }\end{array}$ & $\begin{array}{l}\text { Ab. } \\
\text { émo. }\end{array}$ & $\begin{array}{l}\text { Ab. } \\
\text { phys. }\end{array}$ & Négl. \\
\hline NCPCA & Idem & Idem & 12 mois 1993 & 45 & 15 & 2,6 & 1,1 & 4,1 & 6,8 \\
\hline NCPCA & Idem & Idem & 12 mois 1990 & 40 & -- & -- & -- & -- & -- \\
\hline NCPCA & Idem & Idem & 12 mois 1986 & 33 & -- & -- & -- & -- & -- \\
\hline \multirow[t]{2}{*}{$\begin{array}{l}\text { \#12 U.S. Dept. } \\
\text { (1998) } \\
\text { NCANDS, } \\
\text { États-Unis }\end{array}$} & $\begin{array}{l}\text { Déf. légales } \\
\text { Statistiques } \\
\text { Niveau } 1\end{array}$ & $\begin{array}{l}\text { Oui } \\
\text { partiel }\end{array}$ & $\begin{array}{l}\text { Générale } \\
\text { américaine } \\
\text { Enfants } \\
\text { (0-17 ans) }\end{array}$ & & & & & & \\
\hline & & & 12 mois 1996 & 44 & 15 & 1,8 & 1,0 & 3,6 & 8,0 \\
\hline \#13 NCANDS & Idem & & 12 mois 1993 & 43 & 14 & 2,1 & 0,7 & 3,5 & 7,5 \\
\hline \#14 NCANDS & Idem & & 12 mois 1990 & 41 & 14 & 2,1 & 1,1 & 3,3 & 7,6 \\
\hline \multirow[t]{5}{*}{$\begin{array}{l}\text { \#15 Knudsen } \\
\text { (1988) États-Unis }\end{array}$} & $\begin{array}{l}\text { Déf. légales } \\
\text { Statistiques } \\
\text { Niveau } 1\end{array}$ & Non & $\begin{array}{l}\text { Comté } \\
\text { d'Indiana } \\
\text { Enfants } \\
\text { (0-14 ans) }\end{array}$ & & & & & & \\
\hline & & & 3 ans $-82 / 84$ & 72 & 32 & -- & -- & -- & -- \\
\hline & & & 3 ans $-79 / 81$ & 64 & 24 & -- & -- & -- & -- \\
\hline & & & 4 ans $-74 / 78$ & 44 & 12 & -- & -- & -- & -- \\
\hline & & & 9 ans $-65 / 73$ & 11 & 6 & -- & -- & -- & -- \\
\hline $\begin{array}{l}\text { \#16 White \& } \\
\text { Cornely (1981) } \\
\text { États-Unis }\end{array}$ & $\begin{array}{l}\text { Déf. chercheurs } \\
\text { Questionnaire } \\
\text { Niveau } 2\end{array}$ & Non & $\begin{array}{l}\text { Pop. navajo } \\
\text { Enfants } \\
(0-9 \text { ans) } \\
12 \text { mois } 1975\end{array}$ & -- & 10,3 & -- & -- & 2 & 8,3 \\
\hline $\begin{array}{l}\text { \#17 Trocmé et al. } \\
\text { OIS (1994) } \\
\text { Ontario, Canada }\end{array}$ & $\begin{array}{l}\text { Déf. chercheurs } \\
\text { Questionnaire } \\
\text { Niveau } 1\end{array}$ & $\begin{array}{l}\text { Oui } \\
\text { partiel }\end{array}$ & $\begin{array}{l}\text { Générale } \\
\text { ontarienne } \\
\text { Enfants } \\
(0-15 \text { ans }) \\
3 \text { mois } 1993\end{array}$ & 21,3 & 5,6 & 1,6 & 0,4 & 1,9 & 2,0 \\
\hline $\begin{array}{l}\text { \#18 Trocmé } \\
\text { (1992) } \\
\text { Ontario, Canada }\end{array}$ & $\begin{array}{l}\text { Déf. légales } \\
\text { Statistiques } \\
\text { Niveau } 1\end{array}$ & Non & $\begin{array}{l}\text { Générale } \\
\text { ontarienne } \\
\text { Enfants } \\
\text { ( } 0-15 \text { ans) } \\
12 \text { mois } 1990\end{array}$ & 39 & 17 & 2,8 & 0,2 & 3,2 & 10,8 \\
\hline $\begin{array}{l}\text { \#19 Mayer (1997) } \\
\text { Québec, Canada }\end{array}$ & $\begin{array}{l}\text { Déf. légales } \\
\text { Statistiques } \\
\text { Niveau } 1\end{array}$ & $\begin{array}{l}\text { Oui } \\
\text { partiel }\end{array}$ & $\begin{array}{l}\text { Générale } \\
\text { montréalaise } \\
\text { Enfants } \\
(0-17 \text { ans }) \\
24 \text { mois } 91 / 93\end{array}$ & 9,4 & -- & -- & -- & -- & -- \\
\hline $\begin{array}{l}\text { \#20 Tourigny \& } \\
\text { Bouchard } \\
(1994)^{67} \text { Québec, } \\
\text { Canada }\end{array}$ & $\begin{array}{l}\text { Déf. légales } \\
\text { Dossiers } \\
\text { Niveau } 1\end{array}$ & $\begin{array}{l}\text { Oui } \\
\text { partiel }\end{array}$ & $\begin{array}{l}\text { Générale } \\
\text { montréalaise } \\
\text { Familles } \\
(0-17 \text { ans }) \\
9 \text { mois } 88 / 89\end{array}$ & $\begin{array}{l}10,9 \\
13,1\end{array}$ & $\begin{array}{l}5,4 \\
6,1\end{array}$ & $\begin{array}{l}-- \\
-\end{array}$ & $\begin{array}{l}-- \\
--\end{array}$ & $\begin{array}{l}-- \\
--\end{array}$ & $\begin{array}{l}-- \\
--\end{array}$ \\
\hline $\begin{array}{l}\text { \#21 Chamberland } \\
\text { et al. (1986) } \\
\text { Québec, Canada }\end{array}$ & $\begin{array}{l}\text { Déf. légales } \\
\text { Dossiers } \\
\text { Niveau } 1\end{array}$ & Oui & $\begin{array}{l}\text { Générale } \\
\text { montréalaise } \\
\text { Enfants } \\
(0-17 \text { ans })\end{array}$ & $6,0^{7}$ & -- & -- & -- & -- & -- \\
\hline & & & $\begin{array}{l}\text { Familles } \\
(0-17 \text { ans }) \\
7 \text { mois } 1983\end{array}$ & 5,0 & -- & -- & -- & -- & -- \\
\hline
\end{tabular}




\section{T A B LE A U 1 (Suite)}

Taux d'incidence annuel des signalements pour mauvais traitements et des signalements jugés fondés après évaluation (toutes formes confondues) et taux d'incidence annuel par forme de mauvais traitements

\begin{tabular}{|c|c|c|c|c|c|c|c|c|c|}
\hline \multirow{2}{*}{$\begin{array}{l}\text { \# Études } \\
\begin{array}{c}\text { Auteur/Année/ } \\
\text { Pays }\end{array}\end{array}$} & \multicolumn{2}{|c|}{$\begin{array}{l}\text { Méthodologie/ } \\
\text { Échantillon }{ }^{1}\end{array}$} & \multicolumn{7}{|c|}{ Taux d'incidence annuel (/1000) } \\
\hline & $\begin{array}{l}\text { Définitions } \\
\text { Méthode / Niveau }\end{array}$ & $\begin{array}{l}\text { Contrôle } \\
\text { Duplication }\end{array}$ & $\begin{array}{l}\text { Population } \\
\text { Unité } \\
\text { Période de } \\
\text { collecte }\end{array}$ & Signalée & Fondé $^{2}$ & $\begin{array}{l}\text { Ab. } \\
\text { sex. }\end{array}$ & $\begin{array}{l}\text { Ab. } \\
\text { émo. }\end{array}$ & $\begin{array}{l}\text { Ab. } \\
\text { phys. }\end{array}$ & Négl. \\
\hline $\begin{array}{l}\text { \#22 Guay \& } \\
\text { Lavergne (1998) } \\
\text { Québec, Canada }\end{array}$ & $\begin{array}{l}\text { Déf. chercheurs } \\
\text { Questionnaire } \\
\text { Niv. } 2 \text { (écoles) }\end{array}$ & $\begin{array}{l}\text { Oui } \\
\text { partiel }\end{array}$ & $\begin{array}{l}\text { Générale } \\
\text { écoles primaires } \\
\text { Enfants } \\
(6-14 \text { ans }) \\
8 \text { mois } 1997\end{array}$ & -- & 31,5 & 0,6 & 1,7 & 1,1 & 65,3 \\
\hline $\begin{array}{l}\text { \#23 MSSS } \\
\text { (1997) } \\
\text { Québec, Canada }\end{array}$ & $\begin{array}{l}\text { Déf. légales } \\
\text { Statistiques }\end{array}$ & Non & $\begin{array}{l}\text { Générale } \\
\text { québécoise } \\
\text { Enfants } \\
\text { (0-17 ans) }\end{array}$ & & & & & & \\
\hline MSSS & Niveau 1 & & 12 mois $97-98$ & 10,2 & 6,7 & 0,7 & -- & 1,1 & 4,9 \\
\hline MSSS & & & 12 mois $96-97$ & 9,5 & 6,3 & 0,7 & -- & 1,0 & 4,5 \\
\hline MSSS & & & 12 mois $95-96$ & 9,9 & 6,5 & 0,8 & -- & 1,0 & 4,8 \\
\hline MSSS & & & 12 mois $94-95$ & 9,5 & 6,3 & 0,8 & -- & 0,9 & 4,6 \\
\hline \multirow[t]{5}{*}{$\begin{array}{l}\text { \#24 Wright et al. } \\
\text { (1997) } \\
\text { Québec, Canada }\end{array}$} & $\begin{array}{l}\text { Déf. légales } \\
\text { Statistiques } \\
\text { Niveau } 1\end{array}$ & Non & $\begin{array}{l}\text { Générale } \\
\text { québécoise } \\
\text { Enfants } \\
\text { (0-17 ans) }\end{array}$ & & & & & & \\
\hline & & & 12 mois $95-96$ & -- & -- & 0,9 & -- & -- & -- \\
\hline & & & 12 mois $94-95$ & -- & -- & 0,9 & -- & -- & -- \\
\hline & & & 12 mois $93-94$ & -- & -- & 1,0 & -- & -- & -- \\
\hline & & & 12 mois $92-93$ & -- & -- & 1,4 & -- & -- & -- \\
\hline
\end{tabular}

1. Le lecteur peut se référer à la section Méthodologie pour une description détaillée de la signification des termes utilisés dans le tableau.

2. Le taux d'incidence de cas signalés correspond à l'ensemble des signalements retenus pour une évaluation alors que le taux d'incidence de cas fondés correspond aux situations de mauvais traitements confirmés à la suite de l'évaluation.

3. La loi hollandaise ne rend pas obligatoire le signalement de situations de mauvais traitements envers un enfant, les familles se présentent donc volontairement chez les médecins pour recevoir de l'aide. Cette situation explique, en partie, les faibles taux indiqués par l'étude.

4. Les résultats de la première ligne correspondent aux familles de la communauté de Baltimore et ceux de la seconde aux familles d'accueil de cette région.

5. Les résultats de la première ligne correspondent aux mauvais traitements qui entraînent pour l'enfant des conséquences négatives observables alors que ceux de la seconde ligne correspondent aux mauvais traitements représentant une menace importante pour la santé et la sécurité de l'enfant

6. Les résultats de la première ligne correspondent aux familles canadiennes-françaises vivant sur le territoire de l'île de Montréal alors que ceux de la seconde ligne concernent les familles haïtiennes vivant sur le même territoire. Ces taux incluent les troubles de comportement sérieux. À l'exception du taux d'incidence des signalements retenus pour évaluation, les taux correspondent non pas aux familles pour lesquelles le signalement a été jugé fondé mais aux familles prises en charge par les services de protection.

7. Les taux de ces études incluent les troubles de comportement sérieux. 
contact physique avec le corps de l'enfant (baisers, caresses, masturbation, pénétration, etc.) ou d'autres actes à connotation sexuelle mais sans contact physique tel l'exhibitionnisme (Tourigny, 1991). L'abus émotionnel a trait à des comportements de rejet, d'humiliation, de dépréciation, de cruauté psychologique, de menace, d'intimidation, de «terrorisation » et autres gestes de violence psychologique qui menacent la construction d'un concept de soi positif et cohérent (MSSS, 1991 ; Fortin, 1992 : 5-18). Au-delà de ces grands paramètres, il existe différentes manières de définir et de rendre opérationnelle chacune des formes de mauvais traitements. Plusieurs facteurs peuvent conditionner la définition des mauvais traitements, entre autres les critères légaux en vigueur, les valeurs culturelles et les normes sociales d'une communauté donnée. La variabilité dans les définitions a des conséquences directes sur l'évaluation de l'ampleur du phénomène. Ainsi les écarts entre les taux d'incidence sont-ils plus ou moins importants selon que les définitions des mêmes problématiques sont plus ou moins restrictives. Par exemple, certaines définitions ne couvrent que les situations d'abus et de négligence qui entraînent pour l'enfant des conséquences négatives observables alors que d'autres, en plus d'englober ces situations, couvrent celles qui constituent un danger potentiel pour l'enfant. C'est le cas notamment des définitions utilisées par le NIS aux États-Unis et de certaines définitions légales dont celles retenues par la Loi sur la protection de la jeunesse au Québec. Le contexte dans lequel surviennent les situations de mauvais traitements constitue un autre élément des définitions susceptible de faire varier les taux. Ainsi, certaines définitions ne couvrent que les situations de maltraitance intrafamiliale alors que d'autres visent également les situations d'abus et de négligence envers les enfants qui surviennent hors de la famille. Bon nombre des définitions légales sur lesquelles s'appuient les services de protection pour intervenir, de même que celles qui sont utilisées dans le NIS-1 et le NIS-2, font partie de la première catégorie, alors que celles du NIS-3 tombent dans la seconde catégorie, du moins pour ce qui est de l'abus sexuel. La tranche d'âge des enfants victimes d'abus et de négligence constitue un autre élément de la définition qui peut contribuer à faire varier les taux. L'âge des enfants pris en compte dans les différentes études examinées varie de 0 à 17 ans. Enfin, les formes de mauvais traitements de même que les situations spécifiques à chacune des formes qui sont prises en compte peuvent également varier d'une définition à l'autre. Certaines définitions excluent, par exemple, l'abus émotionnel et la négligence 
éducationnelle. Certaines études américaines réalisées à partir des données des services de protection ne tiennent pas compte de la négligence éducationnelle alors que les données ayant trait à la protection de la banque LPJ au Québec ne permettent pas de documenter l'abus émotionnel. Dans les deux cas, les lois en vigueur ne considèrent pas ces formes de mauvais traitements comme des situations pouvant justifier une intervention de l'État dans la vie des enfants qui en sont victimes. Compte tenu de tous ces éléments, les réalités couvertes sont donc susceptibles de varier d'une étude à l'autre.

Aux fins de la présente analyse, quatre formes de mauvais traitements ont été retenues, à savoir l'abus sexuel, l'abus émotionnel, l'abus physique et la négligence.

\section{Résultats}

Taux d'incidence des mauvais

traitements envers les enfants

Le tableau 1 présente les taux d'incidence des signalements pour mauvais traitements envers les enfants effectués auprès des services de protection de la jeunesse et les taux d'incidence de mauvais traitements confirmés. Ces résultats montrent une variation importante des taux (cas signalés et cas jugés fondés) : les taux d'enfants signalés varient de 5 à 72 pour 1000 alors que les taux d'enfants pour lesquels la situation de mauvais traitement a été jugée fondée varient de 0,2 à 110 pour 1000.

En ce qui concerne chacune des formes de mauvais traitements, les résultats des études recensées montrent que, à l'exception des études australienne et hollandaise, la négligence est nettement la forme de victimisation la plus fréquente : elle représente plus de la moitié des situations de mauvais traitements. Les taux de négligence sont ceux qui varient le plus, soit de 0,2 à 92 enfants négligés pour 1000 enfants. La majorité se situent toutefois entre 1 et 15 enfants négligés pour 1000 . Les enfants victimes d'abus physique représentent environ $20 \%$ des situations de mauvais traitements. La majorité des taux d'incidence se situent entre 1,8 et 6 pour 1000 . Les enfants abusés sexuellement constituent 10 à $12 \%$ des enfants maltraités, les taux variant de 0,3 à 4,5 pour 1000 . Finalement, l'abus émotionnel représente de 5 à $6 \%$ de mauvais traitements envers les enfants et la majorité des taux se situent entre 0,2 et 3 pour 1000 enfants. 
Région

L'examen des taux par région montre des différences importantes : les taux d'incidence d'enfants signalés provenant d'études américaines (de 11 à 72 enfants signalés pour 1000, la majorité se situant entre 30 et 50) sont près de trois fois plus élevés qu'en Australie (entre 11,6 et 13,5 pour 1000 ) et qu'au Québec (5 à 13 pour 1000). Mais ils ne sont que légèrement plus élevés qu'en Ontario (21,3 à 39 pour 1000). Cet écart demeure lorsqu'on examine les taux d'enfants dont la situation de mauvais traitements a été jugée fondée. Les études américaines rapportent des taux variant de 3 à 110 pour 1000 (la majorité se situant entre 15 et 25). L'écart entre les taux provenant des autres études (et donc des autres régions) est toutefois moins important ( 0,2 à 31 enfants maltraités pour 1000). Enfin, à l'exception des taux d'abus émotionnel, les taux par type de mauvais traitements (négligence, abus sexuel et abus physique) suivent la même tendance : les taux sont toujours plus élevés aux États-Unis (et dans une moindre mesure en Ontario) que dans les autres régions. Pour l'abus émotionnel, les statistiques officielles australiennes présentent cependant des taux plus élevés que ceux des ÉtatsUnis et de l'Ontario.

\section{Caractéristiques méthodologiques}

Un aspect pouvant expliquer les variations dans le taux concerne les caractéristiques méthodologiques des études. L'examen de ces caractéristiques montre que deux types d'études d'incidence peuvent être identifiés. Le premier type d'études, de loin le plus fréquent, se caractérise par l'utilisation de définitions légales des mauvais traitements et de données provenant des systèmes d'information des agences de protection de l'enfance (niveau 1), et par l'absence de contrôle concernant la duplication des enfants dans le calcul des taux. Le second type d'études, moins fréquent, se caractérise par l'utilisation de définitions produites par des chercheurs, de questionnaires comme mode de collecte de l'information, de données recueillies auprès des professionnels de la communauté (niveau 2) et, finalement, par des mesures de contrôle à l'égard de la duplication des enfants dans le calcul des taux.

Dans l'ensemble et quelle que soit la forme de mauvais traitements, les études du premier type, c'est-à-dire réalisées à partir des données des agences de protection (niveau 1), rapportent des taux beaucoup moins élevés que les études du second type, c'est-à-dire réalisées à partir de données colligées auprès des professionnels de la communauté 
(niveau 2). Par exemple, les taux des études du NIS-2 et du NIS-3 indiquent qu'entre 15 et 42 enfants américains ont été maltraités pour chaque tranche de 1000, alors que les données provenant des deux autres banques américaines montrent un taux très homogène de 15 enfants maltraités pour 1000 durant des périodes semblables. Les études du NIS sont les seules à documenter le pourcentage d'enfants identifiés à chacun des niveaux. Pour le NIS-3, 927000 enfants (33\%), sur les 2815600 identifiés dans l'enquête, ont fait l'objet d'une évaluation de la part des services de protection. Les taux plus élevés provenant des études de niveau 2 sont principalement attribuables aux situations de négligence et, dans une moindre mesure, aux situations d'abus émotionnel dont les taux augmentent considérablement lorsqu'on passe du niveau 1 au niveau 2. Les professionnels et particulièrement les enseignants semblent donc bien placés pour identifier les situations de négligence.

\section{Évolution des taux}

Le tableau 1 permet également d'examiner l'évolution dans le temps des taux d'incidence fournis par certaines banques de données. Les résultats suggèrent que depuis une vingtaine d'années, les taux d'enfants signalés pour maltraitance ont augmenté de façon importante. Toutefois, cette augmentation semble s'être stabilisée durant les dernières années. Les données du NCPCA montrent que le taux d'enfants signalés est passé de 33 enfants pour 1000 en 1986 à 47 pour 1000 en 1997. Par contre, les données disponibles suggèrent que le taux d'enfants maltraités est stable depuis 1993. De même, l'étude de Knudsen (1988 : 129-143) démontre qu'en 1984, le taux de signalements était 7 fois plus élevé qu'en 1965 alors que le taux d'enfants maltraités avait crû de 5 fois dans la même période. Les données provenant du NCANDS montrent une légère augmentation des signalements et une stabilité des taux d'enfants maltraités depuis le début des années 1990. Enfin, les études du NIS montrent que le taux d'enfants maltraités a plus que doublé entre 1980 et 1993.

Bien que de façon moins prononcée, le même phénomène s'est produit au Québec. Le taux d'enfants signalés et d'enfants maltraités a augmenté considérablement depuis le début des années 1980. Par exemple, Martin (1977) notait qu'entre octobre 1975 et septembre 1976, le Comité de la protection de la jeunesse avait identifié 2216 enfants maltraités. Les données opérationnelles du MSSS montrent que le taux d'enfants maltraités est stable depuis cinq ans, se situant autour de 6,5 (soit 6689 enfants maltraités). 
Concernant l'évolution de chacune des formes de mauvais traitements, les données du NIS montrent que tous les taux augmentent entre 1980 et 1993. L'augmentation est particulièrement importante pour le taux de négligence et celui d'abus sexuel. Par contre, les taux provenant des autres banques de données (NCPCA, NCANDS, MSSS) sont plutôt stables depuis le début des années 1990. Au Québec, à l'exception du taux d'abus sexuel qui diminue légèrement, les autres formes sont très légèrement à la hausse depuis cinq ans. Les données du NCANDS montrent également une très légère augmentation des taux des diverses formes de mauvais traitements à l'exception du taux d'abus sexuel qui diminue très légèrement. Enfin, les données du NCPCA suggèrent que le taux d'abus sexuel et, dans une moindre mesure, ceux reliés à l'abus physique et émotionnel sont à la baisse alors que la négligence est à la hausse.

Taux de cas signalés et taux de cas jugés fondés

La comparaison des taux d'enfants signalés avec les taux d'enfants dont la situation de mauvais traitements a été jugée fondée fournit des renseignements intéressants concernant la proportion d'enfants au sujet desquels le signalement n'a pas été jugé fondé. On constate en effet qu'entre 20 et $76 \%$ des signalements aux agences de protection ne sont pas jugés fondés. Cette proportion varie principalement en fonction des régions considérées. Les études américaines et ontariennes montrent que la proportion de signalements jugés fondés (qui varie de 30 à $45 \%$ selon les études) est moins élevée dans ces deux régions qu'en Australie (soit de 45 à $50 \%$ ) et au Québec (soit de 61 à $76 \%$ ).

\section{Discussion}

Les résultats de cette recension mettent en évidence les variations importantes des taux d'incidence en fonction des régions. Les taux d'incidence sont nettement plus élevés aux États-Unis qu'ailleurs, et ce, bien que des différences interrégionales existent également, quoique à un degré moindre, entre le Québec, l'Ontario et l'Australie. De plus, l'analyse des publications a également mis en évidence des différences de taux à l'intérieur des régions même. Par exemple, les études australiennes montrent que le taux d'incidence varie de $300 \%$ selon les sous-régions examinées (Johnstone et Broadbent, 1998). De même, au Québec, les taux d'incidence peuvent varier de 11 à 37 enfants pour 1000 selon les 
régions administratives (MSSS, 1997). Ces différences importantes sont difficiles à interpréter à partir des données dont nous disposons présentement, les études fournissant très peu d'information sur les principaux aspects pouvant expliquer ces écarts. Parmi les facteurs les plus susceptibles d'expliquer ces variations, notons : 1) l'incidence réelle des situations de victimisation et la présence de facteurs de risque dans une communauté donnée (Trocmé et al., 1995 : 563-586 ; Wright et al., $1997)$; 2) la capacité d'une communauté à identifier les mauvais traitements envers les enfants et sa propension à les signaler (Trocmé et al., 1995 : 563-586) ; 3) les méthodologies ayant mené au taux d'incidence (par exemple, la définition des mauvais traitements, les méthodes de collecte des données, l'unité de mesure, le contrôle des cas dupliqués, etc.) (Trocmé et al., 1995 : 563-586) ; 4) les critères utilisés par les services de protection dans le traitement des signalements ainsi que ceux servant à déterminer si le signalement est fondé ou non (Zellman et Faller, 1996) ; et, finalement, 5) les ressources humaines et financières dont disposent les services de protection pour répondre aux besoins des enfants et de leurs familles (Zellman et Faller, 1996 ; Wright et al., 1997). Il est fort probable que tous ces facteurs interagissent pour mener aux variations constatées entre les taux.

L'examen de l'évolution des taux d'incidence provenant des statistiques officielles des services de protection a également permis de constater que les taux d'enfants signalés ou maltraités sont demeurés relativement stables durant les années 1990, et ce, tant aux États-Unis qu'au Québec. Par contre, les études du NIS démontrent une augmentation constante des taux d'enfants maltraités (par exemple, une augmentation de $67 \%$ du nombre d'enfants identifiés comme victimes d'abus et de négligence entre le NIS-2 et le NIS-3). De plus, le pourcentage d'enfants ayant fait l'objet d'une évaluation de la part des services de protection a diminué significativement au cours de cette même période, passant de 44 à $28 \%$ ou de 51 à $33 \%$ selon le standard de gravité utilisé dans l'étude. En somme, les études du NIS montrent une augmentation des taux d'incidence d'enfants maltraités et une réduction de la proportion d'enfants dont la situation est évaluée par les services de protection.

L'ensemble de ces résultats suggère donc que la stabilité dans les taux d'enfants victimes d'abus et de négligence observée à partir des données produites par les services de protection serait davantage une indication que les services de protection ont atteint un point de saturation quant à leur capacité à répondre à ce problème social que le reflet d'une stabilité 
dans l'incidence réelle des mauvais traitements envers les enfants (Sedlak et Broadhurst, 1996). English (1998: 39-52) souligne d'ailleurs à ce propos que les ressources financières dont disposent les services de protection aux États-Unis n'ont pas augmenté en proportion du nombre croissant de demandes d'aide au cours des années 1990. Pour pallier le manque de ressources, les organismes de protection auraient donc entrepris de resserrer leurs critères de manière à accorder la priorité aux enfants présentant des risques imminents de blessures ou d'atteintes graves. De plus, il est également possible que ce resserrement des critères par les services de protection ait eu pour effet de réduire le nombre de signalements en diminuant la confiance de la communauté et des professionnels dans la capacité des services de protection à répondre adéquatement aux situations d'enfants maltraités. Si cette analyse s'avère exacte, la situation actuelle aurait pour effet de priver de services un nombre de plus en plus important d'enfants maltraités ou à risque de l'être (McCurdy et Daro, 1994 : 75-94 ; English, 1998 : 39-52).

L'examen de l'évolution des taux d'incidence provenant des statistiques officielles montre que la stabilité dans les taux d'enfants maltraités ne reflète pas nécessairement une stabilité dans l'évolution de chacune des formes de mauvais traitements. Au contraire, les résultats suggèrent une diminution du nombre d'enfants abusés sexuellement (bien que les études du NIS démontrent une augmentation), et ce, tant aux États-Unis qu'au Québec, alors que la négligence serait à la hausse. Les hypothèses soulevées pour expliquer l'évolution des taux d'incidence des mauvais traitements peuvent toujours s'appliquer à chacune des formes mais leur importance respective pourrait varier d'une forme à l'autre. Ainsi, le resserrement des critères d'évaluation par les services de protection et le manque de confiance de la communauté pourraient varier en fonction des diverses formes. Par exemple, après avoir analysé l'évolution du taux d'abus sexuel selon les données des services de protection du Québec, Wright et al. (1997) concluaient que des changements majeurs dans les pratiques institutionnelles concernant les abus sexuels étaient survenus et pouvaient expliquer cette diminution. Parmi les changements identifiés, les auteurs rapportaient le fait que les abus sexuels extrafamiliaux n'étaient plus traités par les services de protection et que la réorganisation des services sociaux avait entraîné le démantèlement de certaines équipes spécialisées dans la problématique de l'abus sexuel.

La présente recension a également mis en évidence les limites importantes que comporte l'utilisation des statistiques officielles des agences 
de protection pour la compréhension de la problématique des mauvais traitements envers les enfants, et la nécessité d'améliorer la méthodologie des études pour mieux cerner ce problème social. Que ce soit pour mesurer l'ampleur réelle du phénomène ou pour mieux comprendre les variations entre les taux d'incidence, les taux d'incidence générés à partir des statistiques officielles sont peu utiles. À ce jour, très peu d'études ont été réalisées à partir de données provenant d'autres sources que celles des services de protection. Les prochaines études d'incidence devraient donc : 1) mieux contrôler la production des données à chacune des étapes du processus de recherche ; 2 ) recueillir des données à plus d'un niveau d'identification des situations de mauvais traitements; et 3) procéder à des comparaisons sur les plans régional et international afin de mieux comprendre les variations de taux.

Pour un meilleur contrôle des diverses étapes de la recherche, les chercheurs devraient viser à produire leurs propres définitions plutôt que d'utiliser uniquement celles des services de protection. Par ailleurs, ces définitions devraient tenir compte des situations visées par les lois sur la protection de l'enfance mais également prendre en compte les typologies communément utilisées en recherche, et ce, dans le but de favoriser une meilleure comparabilité des données. Ces définitions devraient également distinguer les différentes formes de mauvais traitements et être basées sur une description détaillée des situations de victimisation (par exemple, les situations spécifiques propres à chaque forme de mauvais traitements, la durée, la fréquence, les caractéristiques du contexte, etc.).

Les recherches futures devraient également utiliser des méthodes de collecte des données reconnues (par exemple, questionnaires administrés aux intervenants, analyse des dossiers, etc.) plutôt que de faire appel aux données produites par les systèmes d'information des services de protection. De cette manière, il serait plus facile pour les chercheurs d'exercer un certain contrôle sur des aspects méthodologiques importants comme l'unité d'analyse servant à calculer les taux d'incidence, la duplication des cas et la question des nouveaux cas, des éléments essentiels du concept d'incidence. Les données des services de protection peuvent s'avérer intéressantes pour dresser un portrait d'ensemble d'un vaste secteur comme celui des services aux enfants en besoin de protection. Toutefois, il convient de rappeler qu'une des caractéristiques importantes de ces données est qu'elles sont de nature administrative. En ce sens, l'objectif poursuivi à travers la production de telles données est d'abord de répondre 
aux besoins de gestion avant de combler ceux de la recherche (Groupe de travail fédéral-provincial sur l'information et sur les services à l'enfance et à la famille, 1994). Aussi apparaitt-il nécessaire d'investir dans le développement de stratégies d'enquête susceptibles de produire des données de qualité et capables de nous permettre de répondre aux questions soulevées par les milieux de recherche et d'intervention concernant le phénomène des mauvais traitements envers les enfants.

La possibilité de documenter plus d'un niveau d'identification à la fois constitue un autre aspect important à prendre en considération dans les prochaines études. Sauf le NIS, aucune étude ne couvre à la fois les niveaux 1 et 2 de la pyramide décrite précédemment. Pourtant, ce genre d'études peut s'avérer intéressant à plusieurs égards. D'abord, elles permettent d'aller au-delà des données des organismes de protection ; elles peuvent fournir un portrait plus complet et plus nuancé de l'incidence et des caractéristiques des situations de mauvais traitements, ce qui favorise une meilleure compréhension des liens qui peuvent exister entre les niveaux de reconnaissance. Ce type d'études présente aussi l'avantage de déterminer le pourcentage d'enfants identifiés comme victimes de mauvais traitements par la communauté des professionnels, qu'ils fassent ou non l'objet d'une évaluation de la part des organismes de protection.

Ces études peuvent également permettre de documenter les pratiques de signalement des diverses catégories de professionnels et contribuer à améliorer leur capacité à identifier les enfants victimes de mauvais traitements ainsi que leur connaissance des situations couvertes par les lois, leur appréciation des faits, leur propension à signaler et les biais potentiels dans leurs signalements aux services de protection (Zellman et Faller, 1996). Les études qui couvrent les deux niveaux à la fois devraient également viser à documenter en détail le processus de décision des services de protection à l'égard des signalements reçus.

Enfin, il est également important de concevoir des études susceptibles de permettre des comparaisons régionales et internationales. Une vaste enquête sur l'incidence et les caractéristiques des situations d'abus et de négligence signalées aux services de protection a présentement cours au Québec (Tourigny et al., 1999) et dans le reste du Canada (Trocmé et al., 1997). Parce qu'il fait appel aux intervenants pour recueillir l'information sur les situations de victimisation portées à l'attention des services de protection et qu'il repose sur un ensemble de définitions uniformes, ce type de stratégie va permettre de procéder à des comparaisons entre 
le Québec et le reste du Canada de même qu'entre les différentes régions du Québec. Ces comparaisons permettront de mieux comprendre les variations dans les taux en documentant la nature des mauvais traitements, les facteurs associés et les différentes pratiques des services de protection.

Cette recension a permis de faire le point sur nos connaissances actuelles concernant l'incidence des mauvais traitements envers les enfants et fait apparaître l'état embryonnaire de la recherche dans ce domaine. Bien que les mauvais traitements envers les enfants soient reconnus comme un problème social important, nous avons encore peu d'information sur l'ampleur et la nature exacte du phénomène, et sur la capacité de nos systèmes de protection et de nos communautés à répondre adéquatement au problème.

\section{Références}

ANGUS, G. et HALL, G. 1996. Child abuse and neglect, Australia 1994-1995, Child Welfare Series, 16. Canberra, Australie : Australian Institute of Health and Welfare.

Benedict, M. I., Zuravin, S., Brandt, D. et AbBey, H. 1994. « Types and frequency of child maltreatment by family foster care providers in an urban population ", Child Abuse and Neglect 18 (7) : 577-585.

Broadbent, A. et Bentley, R. 1997. Child abuse and neglect, Australia, 1995-96, Child Welfare Series, 17. Canberra, Australie : Australian Institute of Health and Welfare.

Chamberland, C., Bouchard, C. et Beaudry, J. 1986. «Les mauvais traitements envers les enfants: réalités canadienne et américaine », Revue canadienne des sciences du comportement $8: 391-412$.

D'ANTONIO, I. J., DARWiSH, A. M. et MCLEAN, M. 1993. « Child maltreatment: International perspectives », Maternal-Child Nursing Journal 21 (2) : 39-52.

Dubowitz, H., Black, M., Starr, Jr. et Zuravin, S. 1993. « A conceptual definition of child neglect », Criminal Justice and Behavior 20 (1) : 8-26.

ENGLISH, D. J. 1998. "The extent and consequences of child maltreatment", The Future of Children 8 (1) : 39-52.

FinKelHOR, D. et DziUbia-Leatherman, J. 1994. «Victimization of children », American Psychologist 49 (3) : 173-183.

FORTIN, A. 1992. «Le mauvais traitement psychologique : une réalité encore mal connue », P.R.I.S.M.E. 3 (1) : 5-18.

Garbarino, J. et Gillian, G. 1980. Understanding abusive families, Lexington, Mass : Lexington Books.

Groupe de travail fédéral-provincial sur l'information et sur les services à l'enfance et à la famille 1994. Rapports annuels sur les services à l'enfance et à la famille, Ottawa : Centre national d'information sur la violence familiale. 
Guay, D. et LAVERGNE, C. 1998. L'incidence des menaces au bien-être des enfants en contexte familial. Rapport de recherche, Montréal, Canada : Centres jeunesse de Montréal.

Johnstone, H. et Broadbent, A. 1998. Child abuse and neglect, Australia 19961997, Child Welfare Series, 21. Canberra, Australie : Australian Institute of Health and Welfare.

KNuDSEN, D. D. 1988. « Child maltreatment over two decades : Change or continuity? », Violence and Victims 3 (2): 129-143.

Larson, O. W., Doris, J. et Alvarez, W. F. 1987. « Child maltreatment among U.S. east coast migrant farm workers », Child Abuse and Neglect 11 : 281-291.

MCCURDY, K. and DARO, D. 1994. « Child maltreatment: A national survey of reports and fatalities », Journal of Interpersonal Violence 9 (1) : 75-94.

MacKenzie, G., Blaney, R., Chivers, A. et Vincent, O. E. 1993. "The incidence of child sexual abuse in Northern Ireland », International Journal of Epidemiology 22 (2) : 299-305.

Martin, G. M. 1977. Étude des caractéristiques des enfants maltraités et des personnes abusives au Québec, Québec : Ministère de la justice, Comité de la protection de la jeunesse.

MAYER, M. 1997. Les contextes d'incidence de mauvais traitements à l'égard des enfants de la région de Montréal, thèse de doctorat, Ph. D. Montréal, Canada: Université de Montréal.

Ministère de la Santé et des Services sociaux - MSSS 1991. Un Québec fou de ses enfants. Rapport du groupe de travail pour les jeunes, Québec: Direction des communications, Ministère de la Santé et des Services sociaux.

Ministère de la Santé et des Services sociaux - MSSS 1995. Cadre normatif CJLPJ : système d'information clientèle des Centres jeunesse, protection de la jeunesse. Banque de données LPJ sur Média, Québec : Gouvernement du Québec.

Ministère de la Santé et des Services sociaux - MSSS 1997. Base de données LPJ sur Média, Québec : Gouvernement du Québec.

National Center on Child Abuse and Neglect 1995. Child maltreatment 1993: Reports from the states to the National Center on Child Abuse and Neglect, Washington, DC: U.S. Department of Health and Human Services.

Pieterse, J. J. et VAN URK, H. 1989. «Maltreatment of children in the Netherlands: An update after ten years », Child Abuse and Neglect 13: 263269.

SEDLAK, A. J. 1991. National incidence and prevalence of child abuse and neglect: 1988 , rapport (version) révisé(e), Rockville : Westat Inc.

SedlaK, A. J. and Broadhurst, D. D. 1996. Executive summary of the national incidence study of child abuse and neglect, Washington, DC: U.S. Department of Health and Human Services, National Center on Child Abuse and Neglect.

Starr, R. H., Dubowitz, H. et Bush, B. A. 1990. « The epidemiology of child maltreatment », p. 23-53 in Children at risk: An evaluation of factors contributing to child abuse and neglect, sous la direction de R. Hammerman et M. Hessen. New York, NY : Plenum Press.

TONMYR, L. 1998. International studies on the incidence and prevalence of child maltreatment: Selected bibliography, Ottawa, Canada: Santé Canada, Child maltreatment division. 
TOURIGNY, M. 1991. Rapport concernant la littérature scientifique sur l'incidence, les facteurs de risque et les programmes de prévention des abus sexuels envers les enfants, document inédit, Groupe de travail pour les jeunes, Québec : Ministère de la Santé et des Services sociaux.

TOURIGNY, M. et BOUCHARD, C. 1994. «Incidence et caractéristiques des signalements d'enfants maltraités : comparaison interculturelle », Child Abuse and Neglect 18 (10) : 797-808.

Tourigny, M., Mayer, M., Wright, J. et LaVergne, C. 1999. Étude d'incidence québécoise (ÉIQL: Devis de recherche, Hull, Canada : Université du Québec à Hull.

TrocmÉ, N. 1992. "Estimating the scope of child abuse and neglect in Ontario: A guide to understanding child maltreatment statistics », Research Connection septembre : 2-6.

Trocmé, N., MCPHEE, D., TAM, K. K. et HAY, T. 1994. Ontario incidence study of reported child abuse and neglect (final report), Toronto, Canada : The Institute for the prevention of child abuse.

Trocmé, N., McPheE, D. et TAM, K. K. 1995. « Child abuse and neglect in Ontario: Incidence and caracteristics », Child Welfare 74 (3) : 563-586.

Trocmé, N., McDonald, L., Hornick, J., Chamberland, C., Mayer, M., Wright, J., Bouchard, C., Burford, G., Sullivan, R. et Boyle, M. 1997. Canadian incidence study of reported child abuse and neglect: Technical and management proposal, Toronto, Canada : University of Toronto, Faculty of Social Work, Centre for Applied Social Research.

U.S. Department of Health and Human Services 1981. National study of the incidence and severity of child abuse and neglect, Washington, DC : Office of Human Development Services, Administration for Children, Youth and Families, Children's bureau, National Center on Child Abuse and Neglect.

U.S. Department of Health and Human Services 1995. Child maltreatment 1993: Reports from the states to the National Child Abuse and Neglect Data System, Washington, DC : U.S. Government Printing Office.

U.S. Department of Health and Human Services, Children's Bureau 1998. Child maltreatment 1996: Reports from the states to the national child abuse and neglect data system, Washington, DC : Government Printing Office.

WANG, C.-T. et DARO, D. 1998. Current trends in child abuse reporting and fatalities: The results of the 1997 annual fifty state survey, Chicago, IL : Center on child abuse prevention research, National committee to prevent child abuse.

White, R. B. et Cornely, D. A. 1981. « Navajo child abuse and neglect study: Comparison group examination of abuse neglect of Navajo children », Child Abuse and Neglect 5 : 9-17.

Wright, J., Boucher, J., Frappier, J.-Y., Lebeau, T. et SAbourin, S. 1997. The incidence of child sexual abuse in Quebec, conférence présenté à la 4th International Family Violence Research Conference, Durham, NH : University of the New Hampshire, $1^{\text {er }}$ juillet.

Zellman, G. L. et Faller, K. C. 1996. «Reporting of child maltreatment », p. 359-381 in The APSAC handbook on child maltreatment, sous la direction de J. Briere, L. Berliner, J. A. Buckley, C. J. et T. Reid. Thousand Oaks, CA : Sage Publications. 